${ }^{1}$ Department of Civil and Environmental Engineering, University of Pittsburgh, Pittsburgh,

Leanne M. Gilbertson ${ }^{1,2^{*}}$, Eva M. Albalghiti ${ }^{2}$, Zachary S. Fishman ${ }^{2}$, François Perreault $^{2,3}$, Charlie Corredor ${ }^{5}$, Jonathan D. Posner ${ }^{5,6}$, Menachem Elimelech ${ }^{2}$, Lisa D. Pfefferle ${ }^{2}$, Julie B. Zimmerman ${ }^{2,4}$

\title{
Shape-Dependent Surface Reactivity and Antimicrobial Activity of Nano-Cupric \\ Oxide
}
${ }^{3}$ School of Sustainable Engineering and the Built Environment, Arizona State University, Tempe, AZ 85287, USA
${ }^{4}$ School of Forestry and Environmental Studies, Yale University, New Haven, CT 06520, USA
${ }^{5}$ Department of Chemical Engineering, University of Washington, Seattle, WA 98195, USA
${ }^{6}$ Department of Mechanical Engineering, University of Washington, Seattle, WA 98195, USA 43 
Crystal Structure Determination by Powder X-Ray Diffraction (XRD).

The XRD measurements were carried out with a Rigaku SmartLab X-ray diffractometer using $\mathrm{Cu} \mathrm{K \alpha}$ radiation $(\lambda=1.5418 \AA$ ) with a rotating anode source, operated at $45 \mathrm{kV}$ and 200 $\mathrm{mA}$.

Morphology and Size via Electron Microscopy.

The micro and nanostructure morphologies of the materials were characterized by Hitachi SU-70 scanning electron microscopy (SEM) and FEI Tecnai Osiris 200kV Tunneling electron microscopy (TEM). Samples were prepared for SEM by depositing dry powder on carbon tape. For TEM, samples were bath sonicated in ethanol, drop cast onto a gold grid, and left to dry. Micrographs were acquired from multiple locations for each sample.

CuO Nanosheet Thickness Measurements via Atomic Force Micrscopy (AFM).

The thickness of $\mathrm{CuO}$ nanosheets was determined using a Bruker Dimension Fastscan Atomic Force Micrometer (AFM). Samples were ultrasonicated in ethanol and drop cast onto a glass slide prior to measurement. The thickness was determined to be $10-20 \mathrm{~nm}$ and associated micrographs are presented in Figure S2.

\section{Surface Area by Brunauer, Emmett and Teller (BET) Method.}

The surface area of all $\mathrm{CuO}$ dry material was measured using a Quanta Chrome Autosorb-1C static volumetric instrument. Samples were degassed at $200^{\circ} \mathrm{C}$ for at least 4 hours and then measured using an eleven point BET protocol. The linear region of the BET plot of the $\mathrm{N}_{2}$ adsorption/desorption isotherm were used to calculate specific surface area. Measurements were repeated in duplicate and reported as an average and standard deviation of the replicate collections.

Dispersed Aggregate Morphology in Experimental Media via Static Light Scattering.

The structural morphology, quantified here as the fractal dimension $\left(D_{f}\right)$, of the dispersed $\mathrm{CuO}$ bulk, nanopowder, and nanosheets was determined using static light scattering (SLS) as previously described. ${ }^{51-53}$ The stock dispersions were prepared by bath sonication $2 \mathrm{mg}$ of each $\mathrm{CuO}$ sample in $10 \mathrm{~mL}$ of DI water (15 min, VWR Model 150T, $135 \mathrm{~W}, 0.2 \mathrm{mg} / \mathrm{mL}$ ). Immediately after sonication, the stock was diluted 10 -fold. SLS data was collected with a multidetector light scattering unit (ALV-GmbH) with a Nd:vanadate laser (Verdi V2, Coherent, Inc., Santa Clara, CA) operating at a wavelength of $532 \mathrm{~nm}$. Multiple iterations were performed for each $\mathrm{CuO}$ sample, correcting the photodetector sensitivity at each iteration, to optimize the linear fit of the data.

Zeta Potential Measurements to Evaluate CuO Surface Charge and Stability in Biological Media and Over a $\mathrm{pH}$ Range.

The surface charge and stability of $\mathrm{CuO}$ bulk, nanopowder, and nanosheets is important for understanding potential interactions with biological organisms, such as gram-negative E.coli used in this study. As such, electrophoretic mobility measurements were collected for each $\mathrm{CuO}$ sample using the NanoBrook Omni (Brookhaven Instruments, NY) with a $659 \mathrm{~nm}$ laser. Samples were prepared in the same manner as for light scattering experiments $(0.2 \mathrm{mg} / \mathrm{mL}, 15 \mathrm{~min}$ 
sonication, 10 -fold dilution) in the biological media $(0.9 \% \mathrm{NaCl})$. The average and standard deviation of the zeta potential (using Smoluchowski analysis type) are determined from 10 runs (30 cycles per run) for each sample.

In addition, electrophoretic mobility measurements were collected over a range of $\mathrm{pH}$ (2 - 9) for the $\mathrm{CuO}$ nanosheets to determine the dependence of material stability as a function of proton concentration of the suspension media. Zeta potential of $\mathrm{CuO}$ nanosheets was determined at a fixed concentration, $20 \mathrm{ppm}$, using dynamic light scattering (DLS) (NICOMP 380 ZLS, Particle Sizing Systems, Santa Barbara, CA) over a 15 min time period. The zeta potentials of particles influence their electrophoretic mobility, as describe by the Henry equation and the Smoluchowski approximation. ${ }^{90}$ All working solutions were prepared in ultrapure water (18.3 $\mathrm{M} \Omega-\mathrm{cm}$, Milli-Q Advantage A10® system, Millipore Corp., Billerica, MA) by first placing the experimental vial on a rotary shaker $(50 \mathrm{rpm})$ for $20 \mathrm{~min}$ at room temperature $\left(23^{\circ} \mathrm{C}\right)$, followed by mild sonication for $1 \mathrm{~h}(40 \mathrm{kHz}, 2510 \mathrm{DTH}$ Branson, Ultrasonic Corp., Danbury, CT) prior to each experiment. A stock $500 \mathrm{mM}$ buffer solutions was prepared for each buffer (e.g., HEPES, citric acid, tris, bis-tris) at $22 \pm 1{ }^{\circ} \mathrm{C}$. All reagents were purchased from Sigma Aldrich. Each stock buffer solution had a $\mathrm{pH}$ near the $\mathrm{pKa}$ of the respective buffer for maximum buffering capacity. ${ }^{91}$ Stock $500 \mathrm{mM}$ buffer solutions were diluted to $20 \mathrm{mM}$ with DI water prior to measuring $\mathrm{pH}$ of the solutions using a dual conductivity and $\mathrm{pH}$ meter.

Inductively Coupled Plasma Mass Spectrometry (ICP-MS).

The presence and concentration of $\mathrm{Cu}^{2+}$ in filtrates were analyzed using ICP-MS (Perkin Elmer DRC-e) with a Scott Crossflow nebulizer and argon plasma. An internal scandium standard was teed into analysis with a glass mixing tee. A certified copper standard at $1000 \mathrm{ppm}$ $( \pm 1 \%$, Fisher Scientific) was used to create a six-point calibration curve. CuO filtrates were diluted 10 -fold and acidified ( $1 \% \mathrm{HNO}_{3}, 10 \mathrm{~mL}$ total volume) prior to data collection. While both ${ }^{63} \mathrm{Cu}$ and ${ }^{65} \mathrm{Cu}$ were measured, ${ }^{63} \mathrm{Cu}$ was used for analysis and there was no significant difference between the trends for each isotope. Each reading included an average of 20 sweeps. The average and standard deviation of three readings was reported. Quality control readings were periodically performed to ensure limited drift during analysis.

Determination of $\mathrm{CuO}$ Interaction with E. coli Cells.

The presence or absence of $\mathrm{CuO}$ materials (bulk, nanopowder, nanosheets) in bacterial cells was evaluated using a combination of transmission electron microscopy (TEM), scanning TEM (STEM), and elemental mapping via electron energy-loss spectrometer. Samples were prepared for imaging by following the method described by Perreault, et al. ${ }^{73}$ Briefly, E. coli were prepared and exposed to the same experimental conditions as described below. After the 1 hour exposure time, a total of $10^{9}$ cells $/ \mathrm{mL}$ for each treatment condition was centrifuged ( $1 \mathrm{~min}$, $5,000 \times \mathrm{g}$ ) to form a visible pellet in a $1.5 \mathrm{~mL}$ Eppendorf tube and fixed in Karnovsky's fixative (2\% paraformaldehyde, $2.5 \%$ glutaraldehyde in $0.2 \mathrm{M}$ Sorenson's buffer, $\mathrm{pH}$ 7.2) overnight at $4^{\circ} \mathrm{C}$. After fixation, the pellet was washed $(3 \times 10 \mathrm{~min})$ with a washing buffer $(0.1 \mathrm{M}$ cacodylate, $\left.0.1 \% \mathrm{CaCl}_{2}, \mathrm{pH} 7.2\right)$ and stained with osmium tetroxide $\left(1 \% \mathrm{OsO}_{4}, 1.5 \% \mathrm{KFeCN}\right.$ in water) for $2 \mathrm{~h}$ at $4^{\circ} \mathrm{C}$. After staining, the pellet was washed with DI water $(3 \times 10 \mathrm{~min})$ and dehydrated in increasing concentrations of acetone (30, 50, 70, 80, 90 and 100\% acetone, $3 \times 10$ min each). Dehydrated samples were infiltrated with resin (EMbed 812, Electron Microscopy Sciences, Hatfield, PA) at room temperature by adding to the pellet solutions of resin:acetone 1:1 (overnight), 2:1 (4 h), 3:1 (overnight), and pure resin (4 h). For pure resin embedding, the sample 
was kept under vacuum to remove air bubbles, and then cured at $58^{\circ} \mathrm{C}$ for $48 \mathrm{~h}$. Embedded samples were cut into $70 \mathrm{~nm}$ slices with a Leica Ultra Cryo UC7 microtome (Leica Mikrosystem GmbH, Vienna, Austria) equipped with a diamond knife (DiATOME, Hatfield, PA) and placed on a gold grid (Holey Carbon, 200 mesh, Electron Microscopy Sciences, Hatfield, PA) for TEM analysis. Samples were visualised with a FEI Tecnai Osiris microscope, operating at an acceleration voltage of $200 \mathrm{kV}$. The nature of the metallic material found inside the cell was

\section{Material Reactivity \\ Electrochemical Activity Assay.}

The $\mathrm{CuO}$ electrodes were scanned at $50 \mathrm{mV} / \mathrm{sec}$ from $-600 \mathrm{mV}$ to $400 \mathrm{mV}$. Impedance measurements were performed using an AC amplitude of $20 \mathrm{mV}$ and a frequencies of 500, 1000, and $2000 \mathrm{hz}$. The flatband potential of a material can be determined using the below equation derived from the imaginary part of the impedance equation

$$
\frac{1}{C^{2}}=\left(\frac{2}{e \epsilon \epsilon_{0} A^{2} N_{D}}\right)\left[\left(V-V_{f b}\right)-\frac{k_{b} T}{e}\right]
$$

where $C$ is the capacitance, $e$ is the charge of an electron, $\varepsilon$ is the dielectric constant of $\mathrm{CuO}, \varepsilon_{o}$ is the permittivity of vacuum, $A$ is the surface area, $N_{D}$ is the charge carrier density, $V$ is the applied voltage, $V_{f b}$ is the flat band potential, $k_{b}$ is Boltzmann's constant, and $T$ is the absolute temperature. By plotting the inverse of the capacitance squared against applied voltage, a line may be extrapolated to the $\mathrm{x}$-axis for the linear portion of the curve. The $\mathrm{x}$-intercept corresponds to $V_{f b}$, the flat band potential.

\section{Catalytic Surface Reactivity Assay.}

It has been shown that metal nanomaterials are catalytically active due to the reduction of their redox potential. ${ }^{92}$ Thus, to act as an effective catalyst for this particular dye-reducing agent pair, the redox potential of $\mathrm{CuO}$ materials must fall between the reduction potential of $\mathrm{MB}$ and $\mathrm{BH}_{4}$ as the reaction is thermodynamically favorable (not kinetically favorable). ${ }^{93}$

CADE consists of final concentrations of $40 \mu \mathrm{M}$ Methylene blue (CAS: 7220-79-3, Sigma-Aldrich, St. Louis, MO), 10 mM Sodium Borohydrate (CAS: 16940-66-2, SigmaAldrich, St. Louis, MO), and 10 mM HEPES (CAS: 7365-45-9 Sigma-Aldrich, St. Louis, MO) at $\mathrm{pH}=7.10 \mathrm{mM}$ HEPES served as the buffering agent to maintain constant $\mathrm{pH}$ throughout the experimental procedures. All working solutions were prepared by dispersing $\mathrm{CuO}$ materials in ultrapure water (18.3 M $\Omega$-cm, Milli-Q Advantage A10® system, Millipore Corp., Billerica, MA) using a rotary shaker $(50 \mathrm{rpm})$ for $20 \mathrm{~min}$ at room temperature $\left(23^{\circ} \mathrm{C}\right)$, followed by mild sonication for $1 \mathrm{~h}$ (40 kHz, 2510DTH Branson, Ultrasonic Corp., Danbury, CT) prior to each experiment. Each experiment began by mixing with a micro magnetic stirring bar, driven by an external magnetic mixer (Cat: H370170, Scienceware, Wayne, NJ). The CuO working solution was mixed with a small aliquot of concentrated $\mathrm{BH}_{4}$ and HEPES solution to reach a final concentration of $10 \mathrm{mM}$ for both reagents. Then, a small volume of concentrated dye was added to a final concentration of $40 \mu \mathrm{M}$ in a $2.5 \mathrm{~mL}$ working volume. CADE's absorbance was continuously monitoring with a portable UV-Vis spectrometer (USB2000+XR1-ES Ocean Optics, Dundin, FL) at the maximum dye's absorbance peak of $\lambda_{\max }=664 \mathrm{~nm}$. CuO catalytic detection studies were preformed in $3 \mathrm{~mL}$ disposable methacrylate cuvette (Cat: 9014, Perfector Scientific, Atascadero, CA) with an optical path length of $1 \mathrm{~cm}$. 
The beta, $\beta$, value was determined in order to quantify differences in surface catalytic activity of the $\mathrm{CuO}$ materials and is the asymptotic constant extracted from equation $4 . \beta$ is determined via the best-fit curve of the plot of the absorbance as a function of time (shown in Figure $1 \mathrm{~b}$ and S5), with an exponential curve, given as

$$
O D=O D_{o} e^{-\frac{t}{\tau}}+\beta
$$

where $O D$ is the optical density of CADE solution, $O D_{o}$ is the optical density at $t=0, \tau$ is the exponential decay rate constant, $t$ is the time transpired from the addition of the $\mathrm{CuO}$ particles, and $\beta$ is a constant that represents the asymptotic $O D$ value. $O D$ data processing and analysis was performed by a custom code written in MATLAB (MathWorks Inc., Natick, MA). We fit $\tau$ and $\beta$ values (41.8 s and 2.2 for the data shown in Figure 1b and S5), which respectively represent the decay time at which the dye absorbance is reduced to 1/e of its initial value and the steady state $O D$ that is reached at long times. $\beta$ values have a dynamic range of 0.01 to 2.8 and potentially serve as a nanoparticle reactivity indicator.

\section{Cytotoxicity Evaluation}

\section{Acellular Assay: CuO-Mediated Glutathione (GSH) Oxidation}

The CuO samples were prepared by dispersing via bath sonication (15 min, VWR Model $150 \mathrm{~T}, 135 \mathrm{~W}, 0.2 \mathrm{mg} / \mathrm{mL}, 10 \mathrm{~mL}$ ) in deionized water. (Note: the sonication time of 15 minutes was determined experimentally as the minimum amount of time required to sufficiently disperse all $\mathrm{CuO}$ materials. Photos of the dispersions after sonication can be found in Figure S7. Experimental solutions were prepared in triplicate and each vial contained the prepared $\mathrm{CuO}$ material, buffer (50 $\mathrm{mM} \mathrm{HCO}_{3}{ }^{-}$, final concentration), and GSH (98\%, ACROS Organics, $33 \mathrm{mM}$ final concentration). The sample vials were rotated constantly throughout the experiment to prevent settling. At specific time points, an aliquot $(0.9 \mathrm{~mL})$ was removed from the experimental solution and added to a solution of Ellman's reagent (5,5'-dithio-bis(2-nitrobenzoic acid), DTNB, $\geq 98 \%$, Sigma-Aldrich, $1.2 \mathrm{mM}$ final concentration) buffered with TRIS-HCl (ultrapure, American Bioanalytical, $\mathrm{pH}=8.3)$. The experimental solution was filtered $(0.22 \mu \mathrm{m}$ syringe filter, PES Whatman), to avoid the potential for confounding interference with the photometric detection. The concentration of GSH in each sample was quantified by measuring the optical density at $412 \mathrm{~nm}\left(\mathrm{OD}_{412}\right)$. Each $\mathrm{CuO}$ sample was compared to the control (no $\mathrm{CuO}$ ) at each time point to obtain a percent oxidation of GSH according to the Beer-Lambert Law:

$$
c=\frac{A}{\varepsilon * l}
$$

where $c$ is the concentration of thiol (M), $A$ is the absorbance at $412 \mathrm{~nm}, \varepsilon$ is the extinction coefficient $\left(14,150 \mathrm{M}^{-1} \mathrm{~cm}^{-1}\right.$ for Ellman's reagent at $\left.412 \mathrm{~nm}\right)$, and $l$ is the path length $(\mathrm{cm})$. 


\section{RESULTS AND DISCUSSION}

\section{Material Characterization}

The crystalline structure of the three $\mathrm{CuO}$ materials was confirmed by X-ray diffraction (XRD). The characteristic diffraction peaks in the XRD spectra (Figure S1d) is signature for cupric oxide, resulting from the (110), (1111)/(002), (111)/(200), (202), (020), (202) lattice plane ${ }^{48}$ Peak broadening in the nanopowder and nanosheet samples, compared to the bulk $\mathrm{CuO}$ sample, is expected for materials containing at least one dimension on the nanoscale. ${ }^{49}$
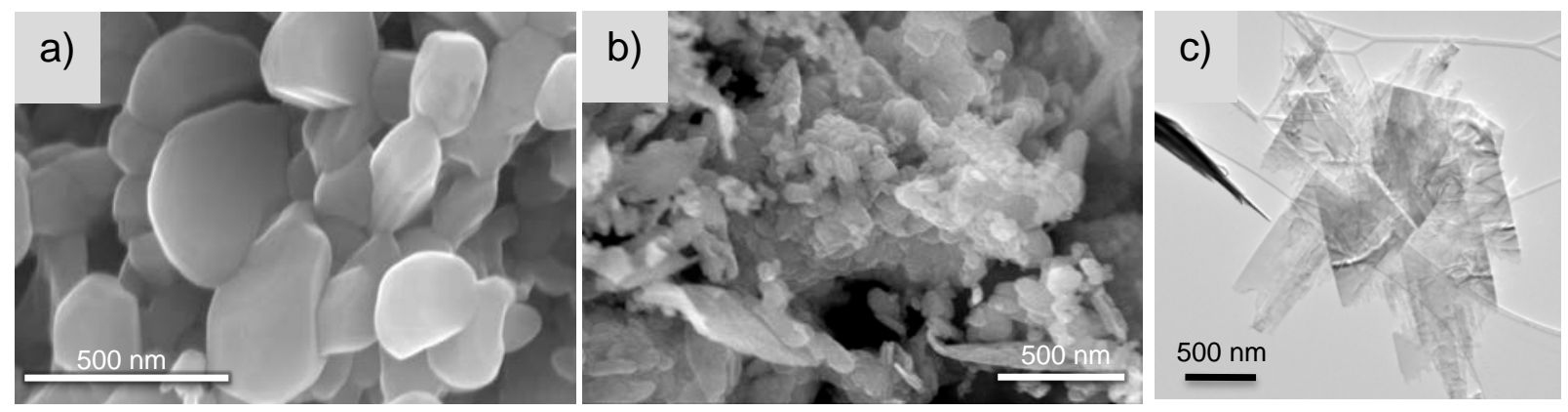

d) 228

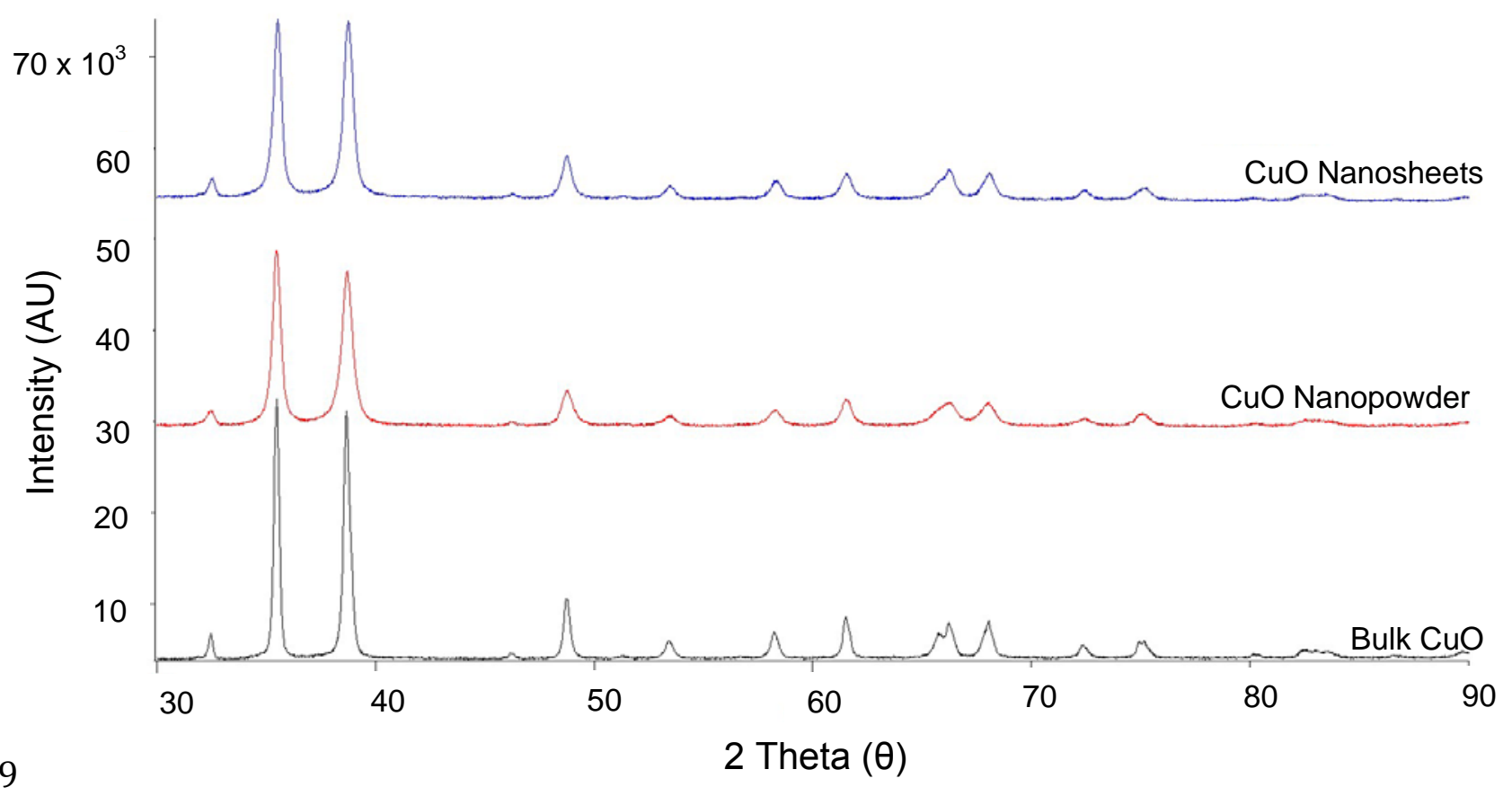

Figure S1. Compiled characterization data for the three copper oxide materials studied. a) SEM micrograph of bulk $\mathrm{CuO}$, b) SEM micrograph of $\mathrm{CuO}$ nanopowder, and c) TEM micrograph of $\mathrm{CuO}$ nanosheets, indicating shape and approximate size of each type of material. d) X-ray diffraction data confirming same chemical composition, 1:1::Cu:O based on the signature crystal structure. 
Morphology, dimensions, and shape were determined using transmission and scanning electron microscopy, TEM and SEM respectively, as well as atomic force microscopy (AFM). Representative TEM (nanosheets) or SEM (bulk and nanopowder) micrographs of the three CuO materials are compiled in Figure S1a-c indicating that bulk $\mathrm{CuO}$ is composed of plate-like particles ( $500 \mathrm{~nm}-3 \mu \mathrm{m}$ ), CuO nanopowder is composed of irregular sphere-like shaped particles $(<50 \mathrm{~nm})$, and $\mathrm{CuO}$ nanosheets are composed sheet-like particles of heterogenous lengths and widths (250 nm $-1 \mu \mathrm{m}$ each). AFM was utilized to determine the thickness of the nanosheets $(10-20 \mathrm{~nm})$ as shown in Figure S2. The size dimensions determined by electron microscopy are associated with single particles of the given $\mathrm{CuO}$ material. Yet, larger aggregates - a compilation of individual particles bound by intermolecular forces (e.g., van der Waals, electrostatic) - are observed in the EM micrographs (Figure S1a-c). While sonication is effective at disaggregating large agglomerates, it is near impossible to obtain stable single $\mathrm{CuO}$ particles without the use of surface functionalization or surfactants, particularly in biological or environmental media. ${ }^{50}$ The focus of this study is on the behavior of the parent material under experimental conditions and therefore, the samples are characterized under these conditions without modification.

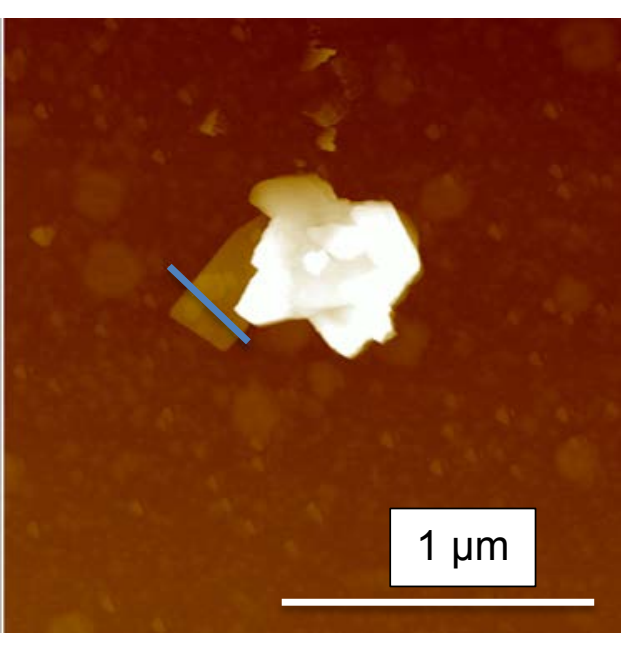

267

268

269

270

271

272

273

274

275

276

277

278

279

280

281 $15 \mathrm{~nm}$ ).
20

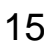

10

5

0

$-5$

$\mathrm{nm}$

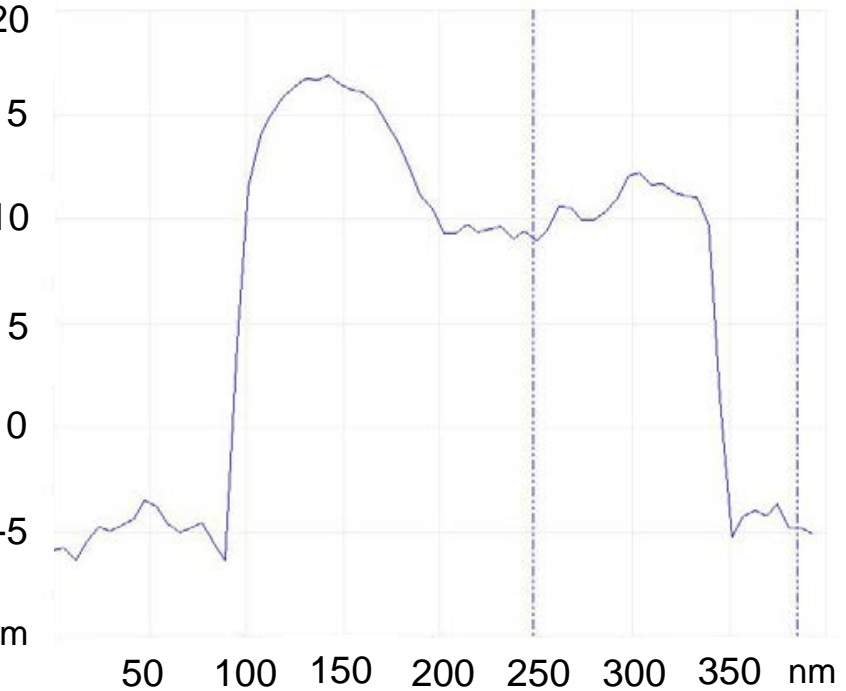

Figure S2. AFM micrograph of CuO Nanosheet and associated sheet thickness (approximately

The relative dispersed aggregate size was determined by dynamic light scattering (DLS). Data for $\mathrm{CuO}$ nanopowder and nanosheets is compiled in Figure S3 as probability distributions of diffusion time $(\tau)$ determined through CONTIN analysis of the compiled raw correlation functions. ${ }^{51}$ Smaller values of $\tau$ are associated with shorter diffusion times and thus, smaller particles or aggregates. Larger values of $\tau$ are associated with longer diffusion times and thus, larger particles or aggregates. Therefore, the location of the probability distributions along the horizontal axis corresponds with the relative aggregate of the dispersed samples. Aggregate size approximations were determined using Stoke-Einstein equation, which requires the spherical particle assumption, and the $\tau$-value is associated with the peak in the distribution curve. ${ }^{51}$ The range in dispersed aggregate size, or polydispersity, is represented by the width of the probability distribution. Here, polydispersity is quantified as the full width at half maximum (FWHM) of the 
probability distribution. ${ }^{51}$ Estimated dispersed aggregate size and size distribution are reported in Table 1 for the $\mathrm{CuO}$ nanopowder and nanosheet samples (data is not reported for the bulk $\mathrm{CuO}$ sample due to instability and significant settling). The dispersed aggregates of the $\mathrm{CuO}$ nanopowder sample are smaller $(\sim 157 \mathrm{~nm})$ and more monodisperse $(\mathrm{FWHM}=888)$ than the nanosheets ( $\sim 557 \mathrm{~nm}$ and FWHM $=2717$, respectively). In addition to the dispersed aggregate size, light scattering was utilized to determine the relative dispersed aggregate morphology via static light scattering (SLS). SLS enables quantification of the fractal dimension $\left(D_{f}\right)-$ a value that indicates whether aggregates are sphere-like $\left(D_{f} \sim 3\right)$, plate-like $\left(D_{f} \sim 2\right)$ or rod-like $\left(D_{f} \sim\right.$ 1). ${ }^{51-53}$ The aggregates of nanopowder, with a $D_{f}=2.1$, are more plate-like in morphology compared to the more rod-like $\mathrm{CuO}$ nanosheets $\left(D_{f}=1.3\right)$.
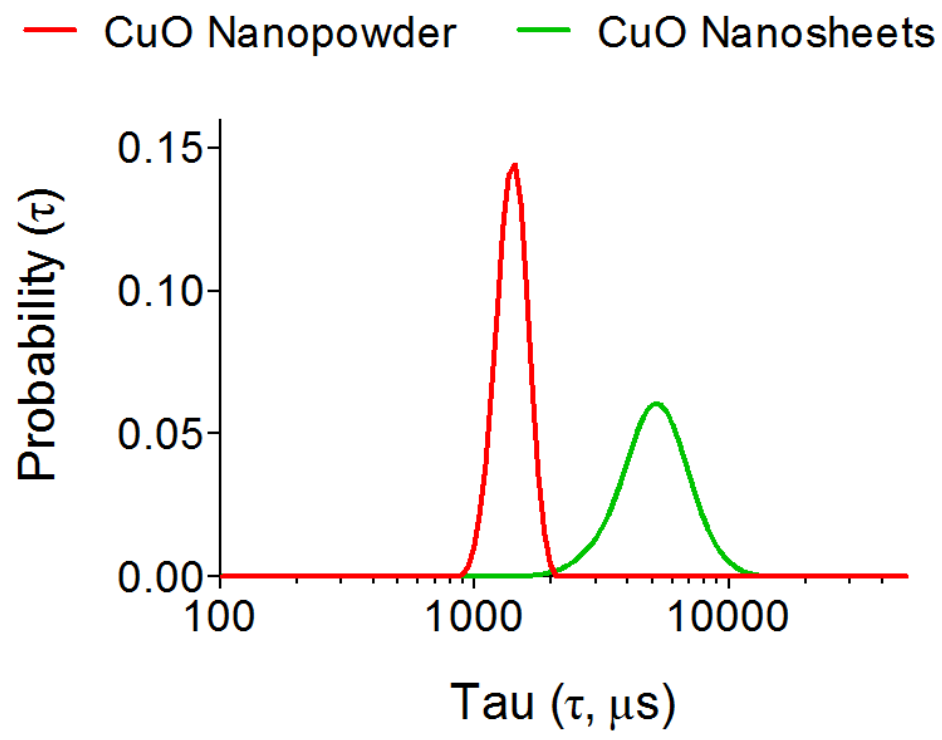

Figure S3. Probability distribution of diffusion time (tau, $\tau$ ) for $\mathrm{CuO}$ nanopowder and nanosheets as determined via dynamic light scattering. Samples were dispersed in biological media $(0.9 \% \mathrm{NaCl}, 0.2 \mathrm{mg} / \mathrm{mL}$ ) and diluted ten-fold prior to sample collection (75 runs, 10 seconds each). The distributions are determined by applying the CONTIN algorithm to the compiled raw correlations functions. Approximate aggregate size is estimated from the peak location and the heterogeneity of aggregate size by the full width at half maximum (FWHM).

Since the surface potential plays an important role in the material-bacteria interactions, electrophoretic mobility measurements were collected to characterize the surface charge of the $\mathrm{CuO}$ samples. Data was collected for each sample in the biological media utilized in the cellular cytotoxicity assay $(0.9 \% \mathrm{NaCl})$ and shows that the $\mathrm{CuO}$ materials are unstable, as indicated by the low magnitude of zeta potential values $(0.05 \pm 8.53$ to $-22.10 \pm 20.91)$. This is to be expected given that the presence of ions is known to significantly influence nanoparticle aggregation. ${ }^{56}$ Similarly, the stability of the $\mathrm{CuO}$ nanosheets over a range in $\mathrm{pH}$ was determined by measuring the electrophoretic mobility between $\mathrm{pH} 2-9$ (Figure S4). The magnitude of material surface charge increased with increasing $\mathrm{pH}$ indicating that $\mathrm{CuO}$ nanosheets are more stable at higher $\mathrm{pH}$. 


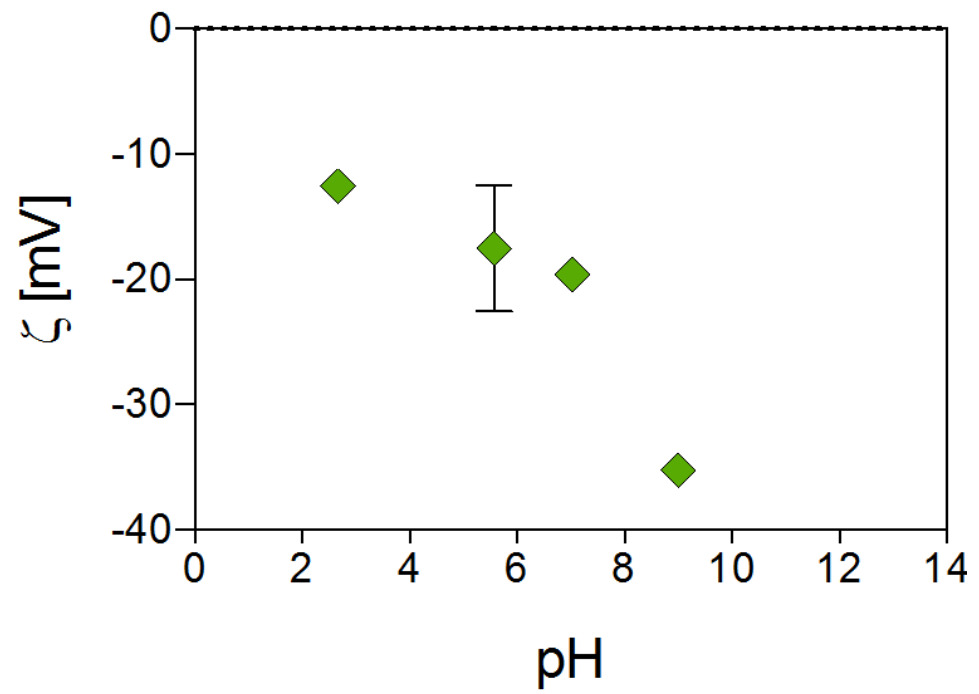

Figure S4. The surface charge of $\mathrm{CuO}$ nanosheets (reported as zeta potential) as a function of $\mathrm{pH}$ at a fixed concentration $(20 \mathrm{ppm})$ and ionic strength $(20 \mathrm{mM})$. The data represents the mean value from a set of six experiments with error bars representing the standard deviation.

a)

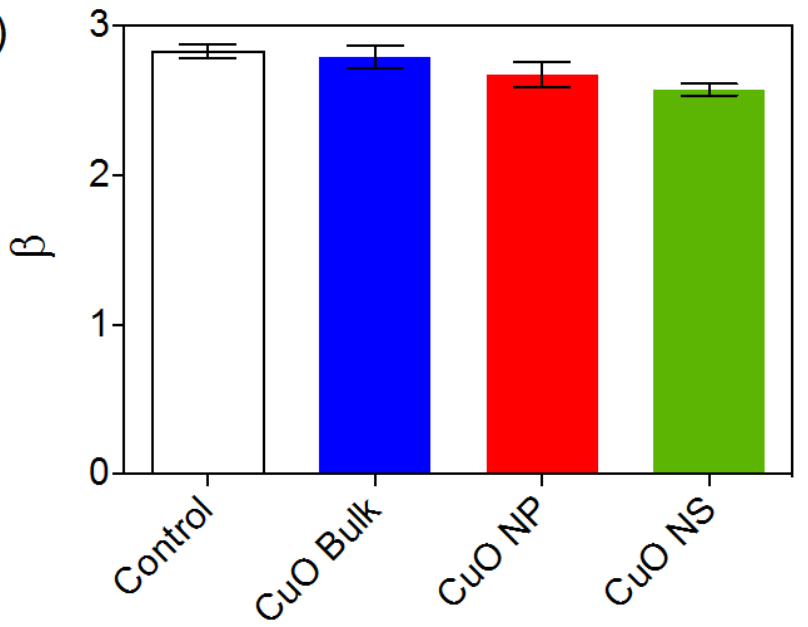

b)

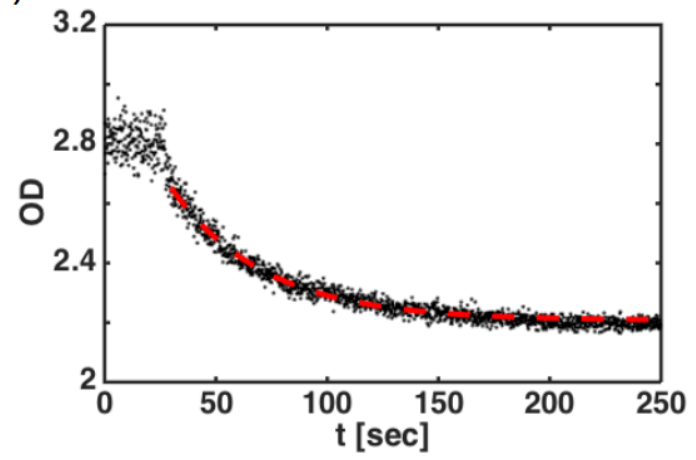

Figure S5. a) $\beta$-values for $\mathrm{CuO}$ bulk, nanopowder and nanosheets at $1 \mathrm{ppm}$. Control experiments correspond with the absence of $\mathrm{CuO}$ material. The lower the $\beta$-value, the higher the material catalytic activity. Each experimental value represents the mean from a set of five experiments with error bars that correspond to the standard deviation. b) $\beta$-values obtained from the fitting equation (Eq 4). ANOVA and subsequent comparison of means test showed that the difference in the values of $\mathrm{CuO}$ nanopowder and nanosheets is statistically significant $(\alpha=0.05$, p-value $<$ 0.0005). 
Glutathione Oxidation

Derivation:

$$
\frac{d A}{d t}=-k A B
$$

The rate of GSH oxidation at any time $t$ is dependent solely upon the concentration of GSH and the concentration of sites of $\mathrm{CuO}$, here represented as a surface area per liter. Solving for $\mathrm{A}$ :

$$
\begin{gathered}
\frac{d A}{d t}=-k A B \rightarrow \frac{d A}{A}=-k B d t \rightarrow \int \frac{1}{A} d A=-k B \int d t \rightarrow \\
\ln A=-k B t+C
\end{gathered}
$$

where $C$ is a constant of integration. It should be noted that for this integration $B$ was held constant, that is the concentration of $\mathrm{CuO}$ sites is constant (according to the assumption that adsorption of GSH and desorption of GSSG from the $\mathrm{CuO}$ surface is fast). If we create a log plot of GSH concentration with respect to time ( $\ln A$ versus $t$ ) we can determine the value of $C$ (by setting $\mathrm{t}=0$, which is $\ln A_{0}$ ), but more importantly we can determine the value of $k$ from the slope.

Data:

\begin{tabular}{|c|c|c|c|c|c|c|}
\hline $\begin{array}{c}\text { CuO } \\
\text { nanopowder }\end{array}$ & 0 min & $15 \mathrm{~min}$ & $30 \mathrm{~min}$ & $45 \mathrm{~min}$ & $60 \mathrm{~min}$ & $120 \mathrm{~min}$ \\
\hline $\begin{array}{c}\text { Mean } \\
\text { \%Oxidation }\end{array}$ & 0.000 & 16.07 & 14.39 & 34.17 & 64.50 & 90.53 \\
\hline $\begin{array}{c}\text { GSH } \\
\text { concentration }\end{array}$ & 3.300 & 2.770 & 2.825 & 2.173 & 1.172 & 0.3124 \\
\hline $\ln (\mathrm{A})$ & 1.194 & 1.019 & 1.039 & 0.7759 & 0.1584 & -1.163 \\
\hline
\end{tabular}

\begin{tabular}{|c|c|c|c|c|c|}
\hline CuO nanosheets & $0 \mathrm{~min}$ & $15 \mathrm{~min}$ & $30 \mathrm{~min}$ & $45 \mathrm{~min}$ & $60 \mathrm{~min}$ \\
\hline $\begin{array}{c}\text { Mean } \\
\text { \%Oxidation }\end{array}$ & 0.000 & 72.28 & 94.34 & 96.66 & 94.80 \\
\hline $\begin{array}{c}\text { GSH } \\
\text { concentration }\end{array}$ & 3.300 & 0.9148 & 0.1867 & 0.1101 & 0.1715 \\
\hline $\ln (\mathrm{A})$ & 1.194 & -0.0890 & -1.678 & -2.207 & -1.763 \\
\hline
\end{tabular}


The second row, mean \%Oxidation, was determined by averaging each column of the data collected. It was then converted to concentrations of GSH using the initial GSH concentration $\left(A_{0}\right)$ using equation (3) below.

$$
\frac{A_{0}-A}{A_{0}} \times 100 \%=\% \text { Oxidation }
$$

Solving for $A$ yields: $A=A_{0}\left(1-\frac{\% \text { oxidation }}{100}\right)$, which is the third row in the table above. The fourth row is the natural log of each cell in the third row.

The plots of $\ln A$ vs $t$ can be found in Figure 6a. Note that the 60-minute time point for the nanosheets was omitted and was considered to be an outlier. The best fit lines drawn here are linear regressions constrained with a y-intercept of 1.19 (the natural log of the initial concentration of glutathione, $\mathrm{A}_{0}$ ). The $\mathrm{R}^{2}$ values are 0.9121 and 0.9645 for the nanoparticles and the nanosheets respectively, but may be increased if the constraint on the y-intercept was relaxed. glutathione oxidation for each the $\mathrm{CuO}$ nanoparticles and the nanosheets.

Nanoparticles:

$$
\ln A=-k B t+C \rightarrow \ln A=-0.0175 t+1.19 \rightarrow-0.0175=-k B
$$

$$
\rightarrow k=9.51 \times 10^{-3} \frac{\text { Liters }}{\text { meters }^{2} \times \text { minutes }}
$$

\section{Nanosheets:}

$$
\ln A=-k B t+C \rightarrow \ln A=-0.0175 t+1.19 \rightarrow-0.0819=-k B
$$

$$
\rightarrow k=20.48 \times 10^{-3} \frac{\text { Liters }}{\text { meters }^{2} \times \text { minutes }}
$$

The concentration of $\mathrm{CuO}$ sites, $B$, was calculated using the mass loading of $\mathrm{CuO}(0.2 \mathrm{mg} / \mathrm{ml}$ in $10 \mathrm{ml})$ and the measured surface area $\left(9.2 \mathrm{~m}^{2} / \mathrm{g}\right.$ and $20.00 \mathrm{~m}^{2} / \mathrm{g}$ for nanoparticles and nanosheets, respectively). The values of $B$ were found to be $1.84 \mathrm{~m}^{2} / \mathrm{L}$ for the nanoparticles and $4.00 \mathrm{~m}^{2} / \mathrm{L}$ for the nanosheets.

\section{Reduction of $\mathrm{CFU} / \mathrm{mL}$}

A: Concentration of living cells $\left(\frac{\text { cells }}{\text { Liter }}\right)$ $B$ : Concentration of $\mathrm{CuO}$ sites $\left(\frac{\text { meters }^{2}}{\text { Liter }}\right)$ $k$ : rate constant $\left(\frac{\text { Liters }}{\text { meters }{ }^{2} \times \text { minutes }}\right)$ \%Reduction: \% Reduction of CFU's (unitless) $A_{0}$ : Initial concentration of living cells $\left(\frac{\text { moles }}{\text { Liter }}\right)$ $t$ : time (minutes) 
As before: $\ln A=-k B t+C$, where $A$ is now the concentration of living cells (cells/L), $k$ is the rate constant for cells dying, $B$ is the concentration of site of $\mathrm{CuO}$ (sites/L), $t$ is time, and $C$ is a

Data:

\begin{tabular}{|c|c|c|c|c|c|c|c|}
\hline $\begin{array}{c}\text { CuO } \\
\text { nanopowder }\end{array}$ & $1.84 \mathrm{E}-03$ & $9.20 \mathrm{E}-04$ & $3.68 \mathrm{E}-04$ & $1.84 \mathrm{E}-04$ & $9.20 \mathrm{E}-05$ & $1.38 \mathrm{E}-03$ & $0.00 \mathrm{E}+00$ \\
\hline $\begin{array}{c}\text { Mean } \\
\text { \%Oxidation }\end{array}$ & $5.71 \mathrm{E}+01$ & $6.90 \mathrm{E}+00$ & $4.08 \mathrm{E}+00$ & $\begin{array}{c}- \\
6.90 \mathrm{E}+00\end{array}$ & $3.51 \mathrm{E}+00$ & $3.26 \mathrm{E}+01$ & $0.00 \mathrm{E}+00$ \\
\hline $\begin{array}{c}\text { Cell } \\
\text { concentration }\end{array}$ & $4.29 \mathrm{E}+08$ & $9.31 \mathrm{E}+08$ & $9.59 \mathrm{E}+08$ & $1.07 \mathrm{E}+09$ & $9.65 \mathrm{E}+08$ & $6.74 \mathrm{E}+08$ & $1.00 \mathrm{E}+09$ \\
\hline $\ln (\mathrm{A})$ & $1.99 \mathrm{E}+01$ & $2.07 \mathrm{E}+01$ & $2.07 \mathrm{E}+01$ & $2.08 \mathrm{E}+01$ & $2.07 \mathrm{E}+01$ & $2.03 \mathrm{E}+01$ & $2.07 \mathrm{E}+01$ \\
\hline
\end{tabular}

431

432

448 Nanoparticles:

$$
k=5.695 \frac{\text { Liters }}{\text { meters }^{2} \times \text { minutes }}
$$

450

451

Also as before:

$$
A=A_{0}\left(1-\frac{\% \text { Reduction }}{100}\right)
$$
divide the slope by $-t$ instead of $-B$.

Nanosheets:

$k=23.73 \frac{\text { Liters }}{\text { meters }^{2} \times \text { minutes }}$

\begin{tabular}{|c|c|c|c|c|c|c|}
\hline CuO nanosheet & $4.00 \mathrm{E}-03$ & $2.00 \mathrm{E}-03$ & $8.00 \mathrm{E}-04$ & $4.00 \mathrm{E}-04$ & $2.00 \mathrm{E}-04$ & $0.00 \mathrm{E}+00$ \\
\hline $\begin{array}{c}\text { Mean } \\
\text { \%Oxidation }\end{array}$ & $1.00 \mathrm{E}+02$ & $9.45 \mathrm{E}+01$ & $7.40 \mathrm{E}+01$ & $3.75 \mathrm{E}+00$ & $1.03 \mathrm{E}+01$ & $0.00 \mathrm{E}+00$ \\
\hline $\begin{array}{c}\text { Cell } \\
\text { concentration }\end{array}$ & $0.00 \mathrm{E}+00$ & $5.54 \mathrm{E}+07$ & $2.60 \mathrm{E}+08$ & $9.63 \mathrm{E}+08$ & $8.97 \mathrm{E}+08$ & $1.00 \mathrm{E}+09$ \\
\hline $\ln (\mathrm{A})$ & $\mathrm{NaN}$ & $1.78 \mathrm{E}+01$ & $1.94 \mathrm{E}+01$ & $2.07 \mathrm{E}+01$ & $2.06 \mathrm{E}+01$ & $2.07 \mathrm{E}+01$ \\
\hline
\end{tabular}

This time, the independent variable is $B$ and not $t$ so the graph must reflect this. The top row are the set of all $B$ values calculated similarly as before using the mass loading per volume of $\mathrm{CuO}$ and the respective specific surface areas. The plot of $\ln A$ vs $B$ is shown in Figure $6 \mathrm{~b}$ for the nanoparticles and the nanosheets. The best fit lines drawn here are linear regressions constrained with a y-intercept of 20.72 (the natural log of the initial cell concentration, $\mathrm{A}_{0}$ ). The $\mathrm{R}^{2}$ values are 0.7825 and 0.9430 for the nanoparticles and the nanosheets respectively, but may be increased if the constraint on the y-intercept was relaxed.

As before, using equation (2) and the slope of the fit lines we can discern the kinetic rate constants of cell death for each the $\mathrm{CuO}$ nanoparticles and the nanosheets. This time, though, we 
453 In this analysis the rate constant for the nanosheets is approximately 4 times that of the 454 nanoparticles, however the $\mathrm{R}^{2}$ value for the best fit line for the nanoparticles is only 0.7825 , 455 which is rather low. In an effort to improve the goodness of fit, a second order model was 456 investigated.

Now, using equation (4) and the slope of the fit lines we can discern the kinetic rate constants of cell death for the second order equations.

This implies that two $\mathrm{CuO}$ sites are required to kill a cell instead of just one. A plot of $\ln A$ against $B^{2}$ is found in Figure 6c. Again, the best fit lines drawn here are linear regressions constrained with a y-intercept of 20.72 (the natural $\log$ of the initial cell concentration, $\mathrm{A}_{0}$ ). The $\mathrm{R}^{2}$ value for the nanoparticles has improved to 0.954 , but has worsened for the nanosheets to 0.8787 (again this would improve slightly with relaxed y-intercept constraint).

472 Nanosheets:

$$
k=12600 \frac{\text { Liters }^{2}}{\text { meters }^{4} \times \text { minutes }^{2}}
$$

474 The units of these rate constants are different than the previous ones because the units of $k$ must make the right hand side of equation (4) dimensionless. Since $B$ is now a squared term, so are its units so the units of $k$ must compensate. For this reason it is unreasonable to compare $k$ constants across models, however comparing within the second order model it appears that $k$ constant for 


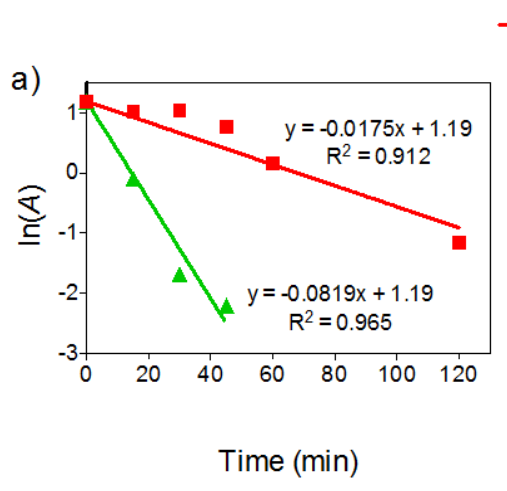

- CuO Nanopowder

b)

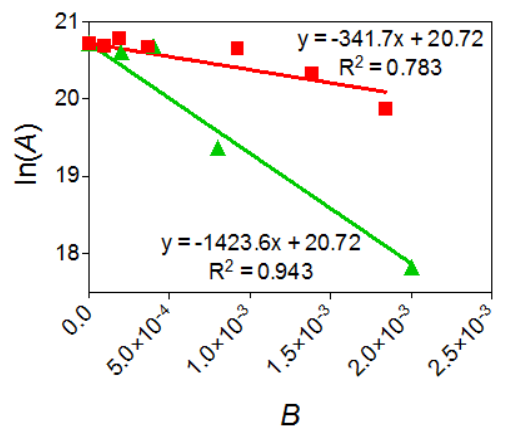

c)

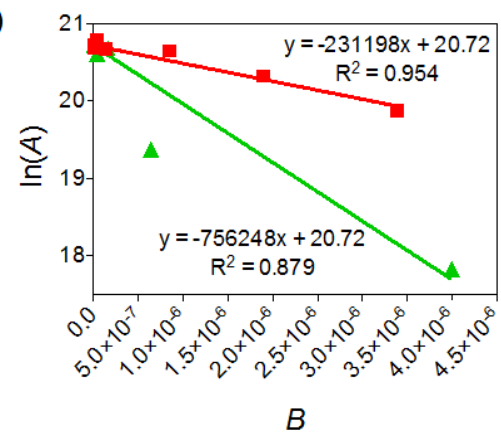

480

481

482

483

484

485

486

487

488

489

490

491

492

493

494

495

496

497

498

499

500

501

502

503

504

505

506

507

508

509

510

511

512

513

514

Figure S6. Plots of the results obtained from the application of kinetic models to the cytotoxicity data for $\mathrm{CuO}$ nanopowder and nanosheets in Figure 2. a) First order kinetic model applied to the GSH oxidation results of $\ln A$ versus time ( $t$ ), where $A$ is the concentration of GSH. b) First order kinetics model applied to the reduction of $\mathrm{CFU} / \mathrm{mL}$ results of $\ln A$ versus the concentration of $\mathrm{CuO}$ sites, $B$, where $A$ is the concentration of living cells. c) Second order kinetics model applied to the reduction of $\mathrm{CFU} / \mathrm{mL}$ results with the variables defined as in $\mathrm{b}$ ). Slopes of the best-fit line provide insight into the rate constant, $k$, for each scenario.
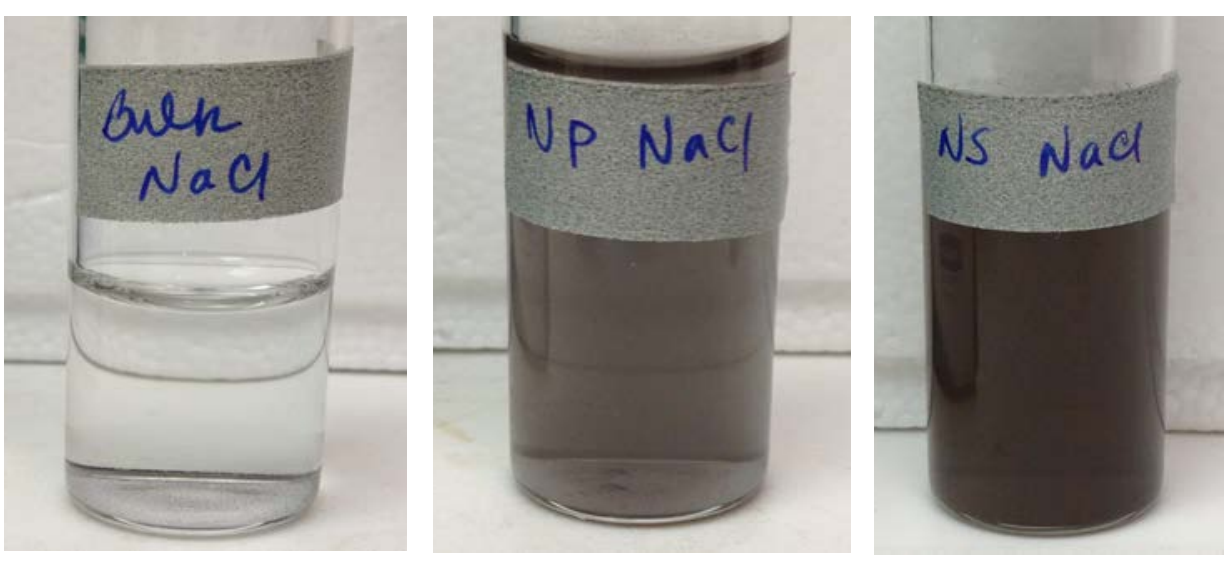

Figure S7. Bulk $\mathrm{CuO}, \mathrm{CuO}$ nanopowder and $\mathrm{CuO}$ nanosheets in $0.9 \% \mathrm{NaCl}$ after 15 minute sonication. 

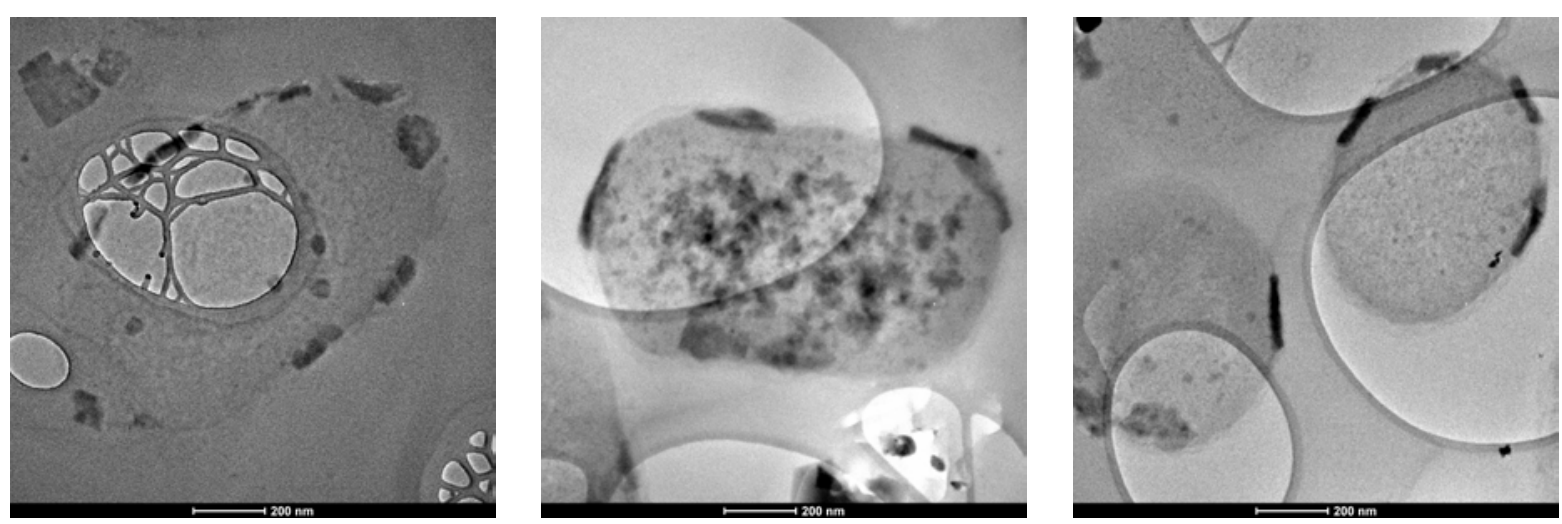

516

517

518

Figure S8. TEM micrographs showing the interaction and accumulation of n-CuO at the cell

519

520

521

522

523
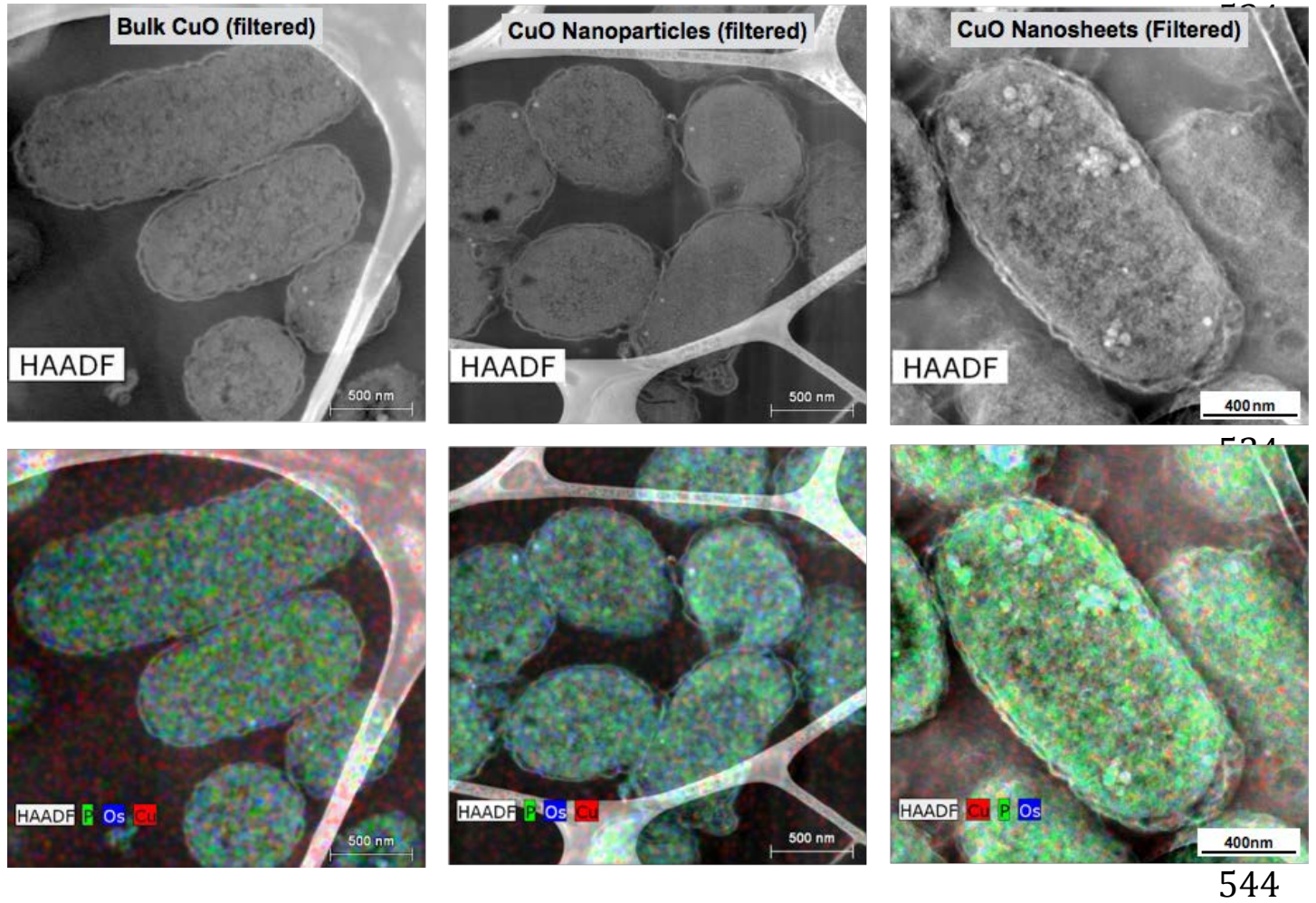

545 Figure S9. STEM-EELS micrographs of E.coli exposed to CuO filtrate showing no indication of 546 intracellular $\mathrm{CuO}$ and confirming the absence of $\mathrm{CuO}$ in the filtered experimental dispersions. 